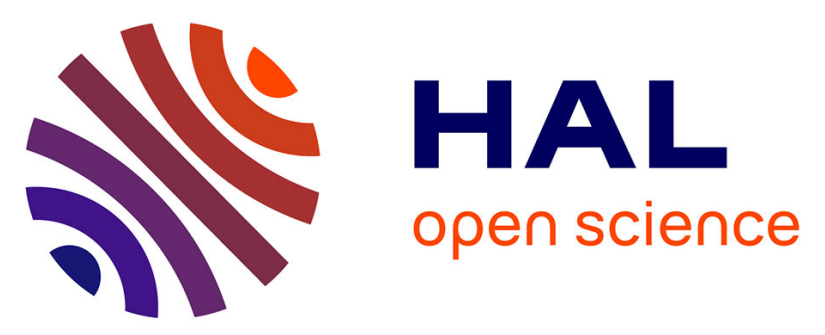

\title{
Mode Stirred Reverberation Chamber (MSRC): a large and efficient tool to lead high frequency bioelectromagnetic in vitro experimentation
}

Sébastien Lalléchère, Sébastien Girard, David Roux, Pierre Bonnet, Françoise Paladian, Alain Vian

\section{To cite this version:}

Sébastien Lalléchère, Sébastien Girard, David Roux, Pierre Bonnet, Françoise Paladian, et al.. Mode Stirred Reverberation Chamber (MSRC): a large and efficient tool to lead high frequency bioelectromagnetic in vitro experimentation. Progress In Electromagnetics Research B, 2010, 26, pp.257-290. 10.2528/PIERB10062313 . hal-01168788

\section{HAL Id: hal-01168788 \\ https://hal.science/hal-01168788}

Submitted on 14 Mar 2019

HAL is a multi-disciplinary open access archive for the deposit and dissemination of scientific research documents, whether they are published or not. The documents may come from teaching and research institutions in France or abroad, or from public or private research centers.
L'archive ouverte pluridisciplinaire HAL, est destinée au dépôt et à la diffusion de documents scientifiques de niveau recherche, publiés ou non, émanant des établissements d'enseignement et de recherche français ou étrangers, des laboratoires publics ou privés.

\section{(ㄷ)(1) $\$$}

Distributed under a Creative Commons Attribution - NonCommerciall 4.0 International 
MODE STIRRED REVERBERATION CHAMBER (MSRC): A LARGE AND EFFICIENT TOOL TO LEAD HIGH FREQUENCY BIOELECTROMAGNETIC IN VITRO EXPERIMENTATION

\author{
S. Lalléchère, S. Girard, D. Roux, P. Bonnet, and F. Paladian \\ Clermont University, Blaise Pascal University \\ BP 10448, F-63000, Clermont-Ferrand \\ LASMEA, CNRS - UMR 6602, F-63173, Aubière, France
}

\begin{abstract}
A. Vian
University of Angers

UMR SAGAH A-462, INRA, Agrocampus Ouest - INHP

2 Bld Lavoisier, F-49045, Angers Cedex 01, France
\end{abstract}

\begin{abstract}
An important aspect of the studies undertaken in bioelectromagnetism relates to the choice of exposure facility. The characteristics of a real electromagnetic environment are far more complex compared to the one plane wave irradiation set-up used in the majority of bioelectromagnetic studies. Moreover, biological requirements should represent the starting point in the design of an in vitro exposure system. Indeed it is important to avoid altering the electromagnetic properties of the exposure system in the presence of biological equipments. Related to these two essential points, this article contributes to show the advantages of a Mode Stirred Reverberation Chamber (MSRC) to guarantee a controlled electromagnetic environment around biological materials for in vitro experimentation. An example of irradiation of in vitro human skin cells cultures is considered to illustrate this paper. In order to show that the biological conditions and the physical requirements for in vitro experiments are checked, two aspects are described. Firstly we achieved the characterization of the electromagnetic field generated around the biological system (both equipments and cultures). Secondly the analysis of the Specific Absorption Rate (SAR) inside the biological medium was evaluated both numerically and experimentally. Initially,
\end{abstract}

Received 23 June 2010, Accepted 29 July 2010, Scheduled 23 October 2010

Corresponding author: S. Lalléchère (sebastien.lallechere@lasmea.univ-bpclermont.fr). 
the statistical properties of fields inside the MSRC were checked with or without the biological devices in order to verify their electromagnetic transparency with respect to the reverberating properties of the electromagnetic environment (inside MSRC). We checked the good agreement of the experimental electromagnetic power distribution with the theoretical one. The computation of electromagnetic energy absorbed by biological medium (SAR) was based upon Finite Difference in Time Domain (FDTD) technique. A numerical analogy was achieved between the MSRC behavior and a free-space finite sum of random plane waves. Simulations are able to provide both an estimation of SAR distribution inside each biological culture dish and a computation of the coupling effects between dishes. Relying on the previous conclusions, temperature measurements were led to evaluate the experimental SAR levels and its time variations inside the MSRC. Two high-frequency $(900 \mathrm{MHz})$ environments were considered: A 10 minutes exposure with field amplitude inside the biological incubator of $7.87 \mathrm{~V} / \mathrm{m}$ and 30 minutes with $41 \mathrm{~V} / \mathrm{m}$ (SAR ranged from $2.6 \mathrm{~mW} / \mathrm{kg}$ to $73 \mathrm{~mW} / \mathrm{kg}$, mean values). Numerical and experimental results prove the ability of MSRC to provide a large and efficient tool to achieve bioelectromagnetic experiments at high frequencies.

\section{INTRODUCTION}

The proliferationof wireless devices including cell phones, base stations, $\mathrm{WiFi}$ and Bluetooth technologies conferred an increasing interest in research programs focusing on the biological effects of high frequency electromagnetic waves in order to establish a formal link between the electromagnetic environment and a biological response. Therefore, the choice of an exposure system arose in the frequency band ranging from $900 \mathrm{MHz}$ to $2.4 \mathrm{GHz}$ (used by wireless communication devices).

\subsection{Exposure Setups for Bioelectromagnetic Experiments in High Frequency Range}

A large number of studies considering the biological and biomedical effects of High Frequency ElectroMagnetic Fields (HF EMF) were carried out starting from test facilities allowing the generation of an electromagnetic plane wave [1]. Review on exposure systems used for bioelectromagnetic experiments in high frequency range [2] enabled to arrange variety of electromagnetic devices according to their major characteristics. First, it was possible to part studies with respect to the experimental protocol (in vitro or in vivo); then systems might be 
classified into various groups with regards to the electromagnetic waves properties and/or interactions (propagating, resonant and radiating).

We could quote for example the anechoic chamber, and also Transverse ElectroMagnetic (TEM) cells [3,4]. TEM cells have been recently used as in vivo exposure tools in bioelectromagnetic experiments such as cerebral changes in rats [5]. Although homogeneity of electric fields was excellent when culture dishes were settled in the centre of the TEM cell, it might be noticed that the electric field quickly decreased in the neighbourhood of the walls. Thus, the ability of TEM cells to perform homogeneous exposure of biological samples is consequently reduced due to the non-uniformity of electric field distributions (quantifying levels of electric fields and dosimetry became complex) [6]. Moreover, the principal disadvantage related to the generation of a plane wave lied in the possible deterioration of its characteristics in the presence of biological equipments. Indeed, in the frequency band considered, the introduction of diffracting elements (biological devices, metallic for instance) into the environment of measurement could disturb the uniformity of the electromagnetic field. In order to create a high amplitude field without powerful amplifiers, specific systems including a metal cavity and electronic cells have previously been designed [7]. The physical properties of this closed structure lead to important amplitudes of the internal field from a wire patch cell combined with an enclosed cavity. A matching device was added to the test cell in order to make it as versatile as possible and usable for different amounts of biological medium. Nevertheless, the cell matching was sensitive to experimental conditions and the induced fields in the biological cell layer and consequently the Specific Absorption Rate (SAR) levels depended highly on the medium quantity. Unlike other exposure systems (efficient from an electromagnetic point of view), the wire patch cell used in this study did not require a complex heat exchanger to ensure a constant temperature. Furthermore some recent works have led to the design of new systems [8,9] respectively to expose rats under farfield conditions (in vivo) or to lead in vivo experiments. Advantages of Radio Frequency (RF) exposure system from [9] relied on its ability to irradiate large cell culture incubator. Although conventional amplifiers might be used to generate low SAR levels of exposure $(0.08 \mathrm{~W} / \mathrm{kg}$ which corresponded to the basic restriction for general public exposure from [10]), bioelectromagnetic experiments needing higher SAR levels might reveal complex. With the idea to exploit directly the modal properties of the oversized metallic cavities, previous studies [11] laid emphasis on the effect of HF EMF on bean root growth. Furthermore, relying on the theory of metallic enclosures, the Mode 
Stirred Reverberation Chamber (MSRC) was used to expose animals to an electromagnetic environment $[12,13]$. Recent works showed great interest in MSRC for the in vivo high frequency electromagnetic exposures of rats and dosimetric studies related [14,15]. For instance, MSRC allowed the use of huge biological devices in a large working volume with a controlled electromagnetic environment (with or without biological material). Although the research programs concerned by in vitro experiments led under investigation various types of exposure systems [16], the MSRC were very seldom used [2]. Previously, we identified a formal link between an electromagnetic environment $(900 \mathrm{MHz})$ and the response of a plant model [17-20] from MSRC experiments. However, the interest of this exposure device for in vitro experiments is highlighted here in the case of human skin cells in culture. Indeed, this model made possible the study of two aspects of the exposure system characterization. The analysis of the properties of the electromagnetic environment in the vicinity of the biological model and that of the power inside the biological culture medium containing the cells allowed an exhaustive study of the MSRC as an exposure system.

\subsection{Brief Overview of MSRC}

The appearance of the MSRC in the literature dates back to the end of the 1960s. The study of the MSRC for applications in electromagnetism developed slowly and had only been introduced in standards in the last twenty years $[21,22]$ for electronic testing processes. A review of the most relevant work done on MSRC investigation until 2002 is given in [23]: as expected, in this article, MSRC were most of the time used for ElectroMagnetic Compatibility (EMC) experiments. Later, [24] showed that MSRC is useful for bioelectromagnetic experiments: This facility was used as the exposure setup for a long-term in vivo study with a large number of unconstrained animals, exposed to radiofrequency electromagnetic fields. The use of MSRC has increased rapidly in the last fifteen years, mainly for EMC testing; many studies have focused on the characterization and measurement of the fields in the chamber $[25,26]$. As explained, the MSRC was classically used for the generation of an electromagnetic field of high amplitude to perform measurements in EMC and also for applications in telecommunications, with, for example, the implementation of wireless devices testing in broad range of multipath radio environment.

In summary, a MSRC is an electrically large, highly conductive, resonant enclosure, where the electromagnetic field structure is continuously altered using paddles or stirrers (except for mode-tuning 

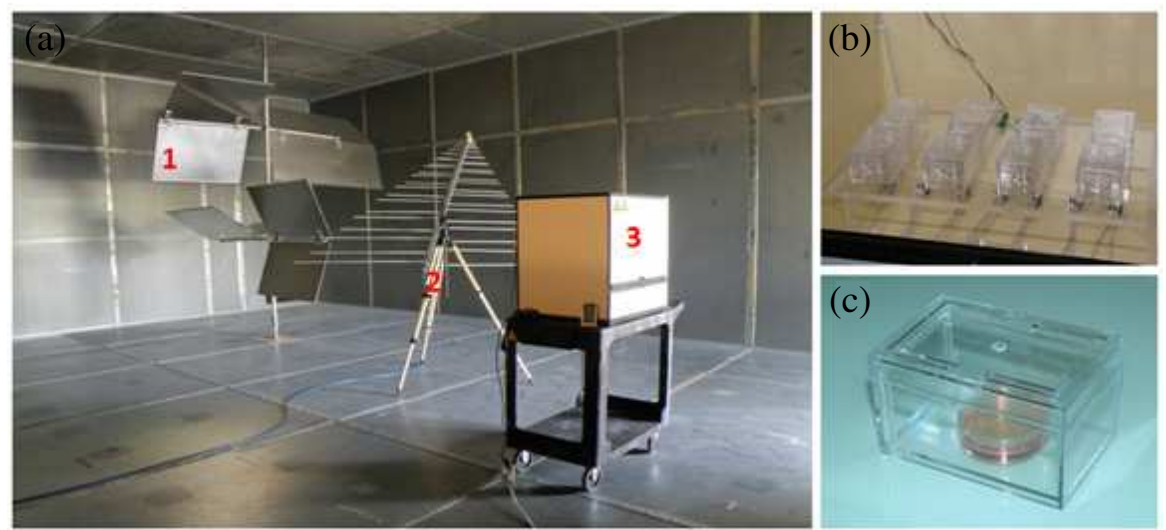

Figure 1. Inner view of LASMEA's MSRC ((a) MSRC with stirrer-1, antenna-2 and culture chamber-3) and biological equipments ((b) plexiglass containers, (c) biological medium) used to expose living cells.

chambers whose working relies on frequency stirring), such that they provide a statistically homogeneous field distribution and isotropic field within a specific volume (called the "working volume"). The typical shape of a MSRC is rectangular (length, width and height should not be simple multiples or rational fractions of each other). Its volume is intimately related to various important factors such as the minimum operating frequency, the number of modes, the quality-factor of the chamber and the working volume (i.e., the domain where the electromagnetic properties of the field are statistically controlled) [28].

According to Fig. 1, the metallic cavity is equipped with a mode stirrer corresponding to a rotating mechanical system (mode-tuning chamber are not considered here). As illustrated by Fig. 1(a)1, this equipment must be as asymmetric as possible, its function during rotation being to modify the internal electromagnetic environment. A stationary electromagnetic field is established when an empty MSRC is excited with a sinusoidal signal at a given frequency. Its spatial distribution corresponds to the superposition of the resonance modes of the cavity. An optimal operation is obtained when overlaps of cavity resonances arose (when the density of modes is sufficiently important, namely at high frequencies). If this condition is verified, and if a rotating stirrer is settled in the cavity, all the components of the electric field at a given spatial point have the same averaged value. Moreover, averaged electric fields at different spatial points may be regarded as identical. These results are obtained over a revolution 
of the stirrer: The measurements of the electric field components or the electromagnetic power lead to determine the statistical parameters of the electromagnetic environment of the MSRC. If the dimensions of the cavity and those of the stirrer are large enough compared to the considered wavelengths, the real and the imaginary parts of the Cartesian components of the electric field follow a normal law, are independent and correspond to the same variance. The modulus of the components of the electric field consequently follows a Rayleigh law. According to this last assumption, it is necessary to avoid direct couplings between the emission and the receiving antennas which is easier to achieve in a MSRC of great dimensions [29]. The statistical data of a MSRC are obtained by performing measurements of electromagnetic field components during a revolution of the stirrer: At a given spatial point of the chamber, those data vary between a minimum and a maximum, but the average value of the field is considered identical from a point to another. A first indicator of the performances of a MSRC is often the value of the standard deviation of the electric field components or of the total electric field [21]. At a given frequency, lower values advocate the right to admit that the density of modes is sufficiently high and the stirrer is sufficiently effective to modify the electromagnetic field from one position to another one. The theoretical laws of probability and the space uniformity of the electric field can be checked from a large amount of measured data. Consequently, in the case of a system that does not modify the electromagnetic environment of the MSRC, its response is independent of its directivity [30], i.e., all parts of the system under test are illuminated by a field with the same characteristics.

\subsection{Biological Experimental Setup}

To illustrate, the present work considers the irradiation of in vitro human skin cells cultures. Data are taken from a recent program [31] developed by our laboratory which aimed to analyze the non-thermal effects of high frequency electromagnetic fields on human skin cells (keratinocytes). The Fig. 1 illustrates the experimental configuration studied here: One cells culture consists of a small dish (cylindrical shape of $41 \mathrm{~mm} \times 12 \mathrm{~mm}$ ) containing $2 \mathrm{~mL}$ of growing medium (pink liquid). Each culture was enclosed in a plexiglass box for maintaining a $\mathrm{CO}_{2}(5 \%)$ atmosphere. Four to height cells cultures were transferred inside a custom-made $(8 \mathrm{~mm}$ thick glass internally insulated with $20 \mathrm{~mm}$ high-density polystyrene) heating incubator $\left(37^{\circ} \mathrm{C}\right)$. These dishes were placed on a $1 \mathrm{~cm}$ width plexiglass board located $16 \mathrm{~cm}$ $(\approx 0.5 \lambda$ at $f=900 \mathrm{MHz}$ ) above the heating device and $26 \mathrm{~cm}$ below the air fan. This culture chamber was then placed inside the working 
volume of the MSRC for irradiation. Two power levels have been assayed respectively corresponding to two characteristic electric fields (see relation 2) surrounding biological media: $\stackrel{\leftrightarrow}{E}_{c}^{\max }=7.87 \mathrm{~V} / \mathrm{m}$ and $41 \mathrm{~V} / \mathrm{m}$ at $900 \mathrm{MHz}$.

As explained in [32], the key issues gathering biological questions and exposure requirements are to design and control the electromagnetic properties of the exposure system. Thus, the fundamental steps are the choice of a proper EM structure, its sizing considering theoretical principles, numerical design and computation of dosimetry. Of course, the last step relies on experimental dosimetry in order to verify numerical results. In this study, results obtained at $900 \mathrm{MHz}$ included both experimental and numerical achievements: First, we obtained a complementary knowledge concerning the electromagnetic environment parameters in the neighbourhood and into the human cells cultures exposed to HF EMF; then, we determined the power absorbed into the biological liquid containing cells.

\section{CONTROL OF THE STATISTICAL CHARACTERISTICS OF THE EXPOSURE SYSTEM}

First, relying on the statistical working of MSRC, it was necessary to define criteria and quantities to check the expected electromagnetic behavior of our MSRC. Then, we put the focus on statistical properties of the empty (without biological devices) MSRC. Finally, we were interested in validating the electromagnetic properties of electric field and power in the vicinity of the biological media (inside the culture chamber, Fig. 1(a)3).

\subsection{Definition of Electromagnetic Validating Criteria}

The interest of the standards [21] and [22] used in MSRC working was principally to provide accurate and robust characteristics to ensure the reproducibility of the tests. This part is dedicated to the definition of electromagnetic notations which were necessary to set the levels of the electric fields obtained in the MSRC. Considering the properties of the electromagnetic environment inside the MSRC, its characterization needed relevant parameters.

The measurement of one component $E_{r}(r=x, y, z)$ for location $i$ and stirrer step $j$ was noted $\left(E_{r}\right)_{i, j}$. Moreover, notations $\leftrightarrow$ and - stand respectively for normalization over one revolution of the stirrer based upon maximizing and averaging. Last, brackets represented averaging over measurement locations. 
The standard [21] enabled to define, for each Cartesian component $r(r=x, y, z)$, a quantity at the location $i$ as follows:

$$
\left(\stackrel{\leftrightarrow}{E}_{r}^{\max }\right)_{i}=\max _{j}\left(\left(E_{r}\right)_{i, j}\right)
$$

which represented the maximum value of electric field component $r$ over a revolution of the stirrer. From [21], electric field might be normalized by incident power $P_{i n}$. In the following, a measured electric field was always given with its corresponding $P_{i n}$.

Thus, it is possible to define a characteristic electric field for each sampled frequency from averaging over $N$ measurement points

$$
\left\langle\stackrel{\leftrightarrow}{E}_{c}^{\max }\right\rangle_{3 N}=\frac{1}{3} \sum_{r=x, y, z}\left\langle\stackrel{\leftrightarrow}{E}_{r}^{\max }\right\rangle_{N}
$$

with

$$
\left\langle\stackrel{\leftrightarrow}{E}_{r}^{\max }\right\rangle_{N}=\frac{1}{N} \sum_{i=1}^{N}\left(\stackrel{\leftrightarrow}{E}_{r}^{\max }\right)_{i}
$$

In our conditions, considering the working frequency (around $900 \mathrm{MHz}$ ), and the variety of tests achieved (with or without biological equipments), various numbers of locations were used to measure electric field. In the following, we referred to the electric field defined by relation (2) only with $\stackrel{\leftrightarrow}{E}_{c}$.

In contrast to [21], the standard [22] was useful only for step by step measurements (not for mode stirring operation). In the following, the number of measurement points was settled to nine (the eight corners of the working volume and its center point). This standard enabled to define a new parameter similar to a total electric field entity (contrary to relation (2) standing for a mean electric field component) averaged over $N$ points

$$
\left\langle\stackrel{\leftrightarrow}{E_{\text {tot }}}\right\rangle_{N}=\frac{1}{N} \sum_{i=1}^{N} \max _{j}\left(\sqrt{\left(E_{x}\right)_{i, j}^{2}+\left(E_{y}\right)_{i, j}^{2}+\left(E_{z}\right)_{i, j}^{2}}\right)
$$

Similarly to previous field $\stackrel{\leftrightarrow}{E}_{c}$ max , the number of measurement points might vary from one configuration to another. Thus, the relation (4) was used for two distinct setups using respectively nine locations (calibration for empty MSRC) and four points (in the presence of the biological incubator). Consequently, it also appeared more convenient to refer indifferently to $\stackrel{\leftrightarrow}{E}_{\text {tot }}$ using quantity in (4) defined for $N=9$ or $N=4$. 
The purpose of this part was not to refer exactly to standards $[21,22]$ but, as previously explained, to define the quantities used here to characterize the levels of the inner electric field (MSRC). Considering the total or the Cartesian component of the electric field, differences appeared and led to the definitions of $\stackrel{\leftrightarrow}{E_{c}}$ and $\overleftrightarrow{E_{t o t}}$. From a physical point of view, we noted that the value given by $\leftrightarrow \max$

$E_{c} \quad$ appeared more penalizing than considering total electric field (maximizing data from all Cartesian components over a whole rotation of the stirrer) and set a maximum level of electric field illuminating biological medium. That was the reason why, in the following, the characteristic levels for electromagnetic exposures were defined from $\leftrightarrow$ max $\stackrel{\leftrightarrow}{E_{c}}$

Finally, from previous electronic criteria, a third parameter for electric field has been defined. From relations (2) and (4), entities defined enable to take into account the electric field maxima. Based upon specificities of MSRC measurements achieved in this article (biological material), the use of maxima components did not reveal inevitably pertinent to dosimetric works. Although previous criteria were well known and largely spread in EMC community and gathering both physical and biological point of view, it appeared interesting for the following treatments to define an averaged total electric entity as follows:

$$
\bar{E}_{\text {tot }}^{\text {moy }}=\frac{1}{180} \sum_{j=1}^{180}\left(\sqrt{\left(E_{x}\right)_{1, j}^{2}+\left(E_{y}\right)_{1, j}^{2}+\left(E_{z}\right)_{1, j}^{2}}\right)
$$

with $\bar{E}_{\text {tot }}^{\text {moy }}$ the averaged value of the total electric field measured at the centre point inside the working volume ( $j$ referring to stirrer step). Previous mean was obtained from 180 steps of mechanical stirrer. The electric field given by (5) was particularly useful for dosimetric part presented in the last section. Moreover, the measurements achieved to compute the quantity given by (4) allowed checking the time stability of electric field in the next part.

\subsection{Statistical Properties of the LASMEA's MSRC}

Let us consider the LASMEA's MSRC whose dimensions are equal to $8.40 \times 6.70 \times 3.50 \mathrm{~m}^{3}$, respectively for the length, width and height. So, the large dimensions of the structure enables to avoid the electromagnetic couplings between the source antenna (log-periodical antenna ETS Lindgren, ref. 3115, 1-18 GHz), directed in the corner of the chamber and the reception equipments. The working volume where the electromagnetic field can be considered as statistically uniform is 


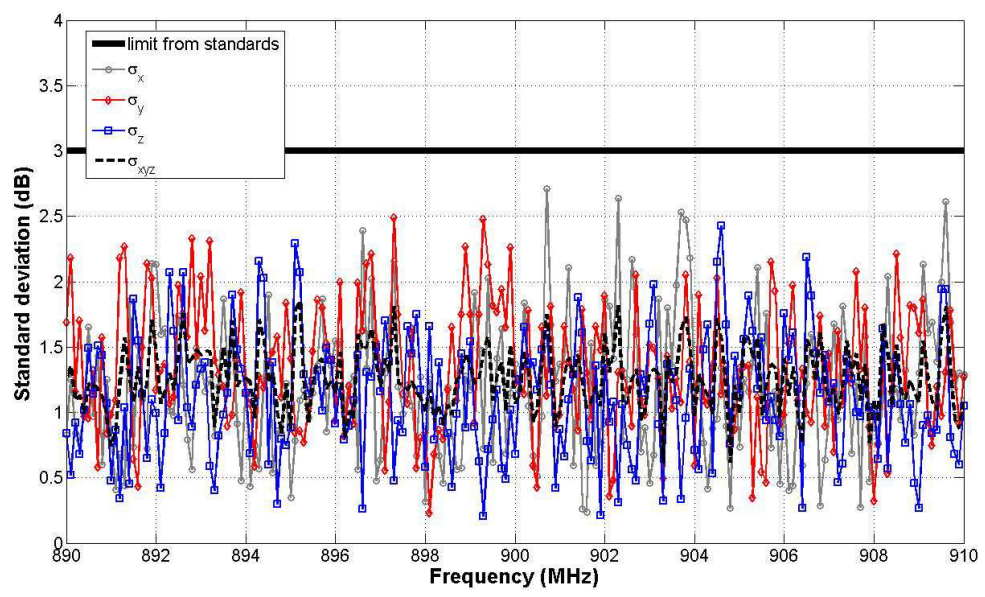

Figure 2. Standard deviation of electric field inside the empty MSRC.

located at $\lambda / 2=c /(2 f)$ of walls and of the stirrer (speed of light, $c=3.10^{8} \mathrm{~m} / \mathrm{s}$; frequency $f$ in $\mathrm{Hz}$ ). The working volume inside the MSRC shapes a $4.20 \times 3.15 \times 0.60 \mathrm{~m}^{3}$ parallelepiped. The electric field was measured by an isotropic probe (Ref. PMM-EP183) allowing the data acquisition of the three rectangular components of the electric field.

Firstly, measurements were carried out in eight space points, i.e., at the tops of a parallelepiped corresponding to the working volume of the MSRC [21]. In the frequency band [890;910 MHz], we verified (Fig. 2) that the values of the standard deviation of the electric field Cartesian components, noted $\sigma_{x}, \sigma_{y}$ and $\sigma_{z}$, as well as that of the standard deviation of the total field, noted $\sigma_{x y z}$ were below the limit of $3 \mathrm{~dB}$, fixed by [21].

In order to confirm these results showing the uniformity of the electric field in the working volume of MSRC, we proceeded to the comparison of the theoretical and experimental distribution laws by means of the adequacy statistical test of Kolmogorov-Smirnov (KS), chosen because of its effectiveness. This test was applied to the independent samples resulting from 500 measurements of the power received by an antenna (the same one as the source antenna) located at the centre of the working volume, the stirrer being continuously in rotation. These measurements were carried out in the range $[890 \mathrm{MHz}$, $910 \mathrm{MHz}$ ] with a step frequency of $\Delta f=0.1 \mathrm{MHz}$. The Fig. 3 represents the maximum value of the difference between the theoretical and experimental distribution functions of the power standardized to 
its mean value on a revolution of the stirrer. It should be noted that only the independent samples are retained for the application of the statistical test for each frequency.

The number of independent samples $N_{\text {ind }}$ is calculated with the classical autocorrelation function method [27]. The offset $\Delta$, i.e., the number of samples at which the correlation coefficient is dropped to $1 / e$ is found, and we have: $N_{i n d}=N_{\text {mes }} / \Delta$. At the frequency of interest, $N_{\text {ind }}$ (equal to 200 at $900 \mathrm{MHz}$ ) is high enough to provide significantly results from the statistical tests. At the frequency of $900 \mathrm{MHz}$, this distance remains lower than the limit given by the Tables [33] for various levels: $\alpha=1 \%, 5 \%$ and $10 \%$. For lower frequencies where the chamber and the stirrer are not electrically large and where the number of excited modes is low, the property of the electromagnetic field uniformity on a revolution of the stirrer is not observed.

Let us recall that $\alpha$ represents the error percentage of rejection of a true assumption. Thus at $900 \mathrm{MHz}$ one observes a non rejection of the theoretical distribution law of the power received by the reception antenna, allowing concluding on the uniformity of the electromagnetic field at this frequency. Thus, a system placed in the working volume of the MSRC is irradiated by an infinite number of plane waves of random polarization and incidence [30]. The question now is to know if the biological samples (human cells) are immersed in an environment whose properties are similar to those of the empty exposure system (MSRC).

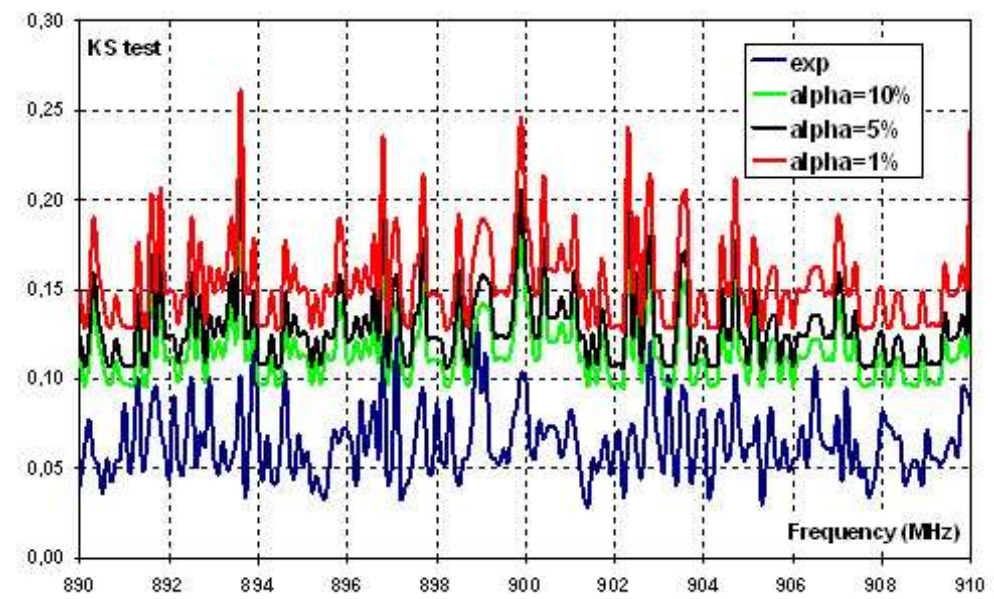

Figure 3. Statistical KS tests for the empty MSRC. 


\subsection{Characterization of the Electromagnetic Environment around the Biological Media}

In this part, we proposed an accurate overview of electromagnetic field characteristics around the biological cultures. To briefly sum up, because of the asymmetry of MSRC stirrer, there were variations in the electric field during each of the $9 \mathrm{~s}$ rotation (Fig. 1(a)1). However, this asymmetric structure ensured that the mean value of the electric field was uniform for each revolution. The electromagnetic environment surrounding the human cells has the following characteristics:

- non-thermal electromagnetic field (two exposure levels: $\stackrel{\leftrightarrow}{E}_{c}^{\max }=$ $7.87 \mathrm{~V} / \mathrm{m}$ and $\underset{\leftrightarrow}{\stackrel{\leftrightarrow}{m}_{c}}=41 \mathrm{~V} / \mathrm{m}$ in the working volume of the MSRC, where $E_{c}$ was given by (2) and (3) according to the number of measurements points,

- high frequency $(900 \mathrm{MHz})$,

- statistical homogeneity, isotropy and uniformity of the electric field over each revolution of the stirrer.

As for the empty chamber, one might consider that the biological samples were irradiated by an infinite number of electromagnetic plane waves characterized by random parameters [30].

Tested human cells were located in a biological medium distributed inside dishes with a diameter of $41 \mathrm{~mm}$ and height of $12 \mathrm{~mm}$, themselves placed in parallelepiped plexiglass containers (Fig. 1(b) and Fig. 1(c)). As depicted in Fig. 1, theses dishes were inserted in an incubator (Fig. 1(a)3) whose sizes were $0.62 \times 0.61 \times 0.46 \mathrm{~m}^{3}$. It was embedded with electrical devices enabling to maintain inner conditions $\left(\mathrm{CO}_{2}\right.$ atmosphere, temperature).

First, we studied the impact of the incubator on the electromagnetic field properties by comparison to the characteristics of the environment inside the empty MSRC. Fig. 4 stands for variations of the loading factor $C L F[21]$ for a frequency $f$ defined as follows:

$$
C L F(f)=\frac{\left\langle P_{r}^{\text {load }}(f)\right\rangle}{\left\langle P_{r}^{\text {empty }}(f)\right\rangle}
$$

where $\left\langle P_{r}^{\text {load }}(f)\right\rangle$ and $\left\langle P_{r}^{\text {empty }}(f)\right\rangle$ stand for the average power measured over a whole stirrer revolution by a reception antenna and a spectrum analyser (ANRITSU MS $2663,9 \mathrm{kHz}-8.1 \mathrm{GHz}$ ) inside the working volume of the MSRC respectively with and without incubator. These measurements have been performed with a frequency step of $100 \mathrm{kHz}$. 


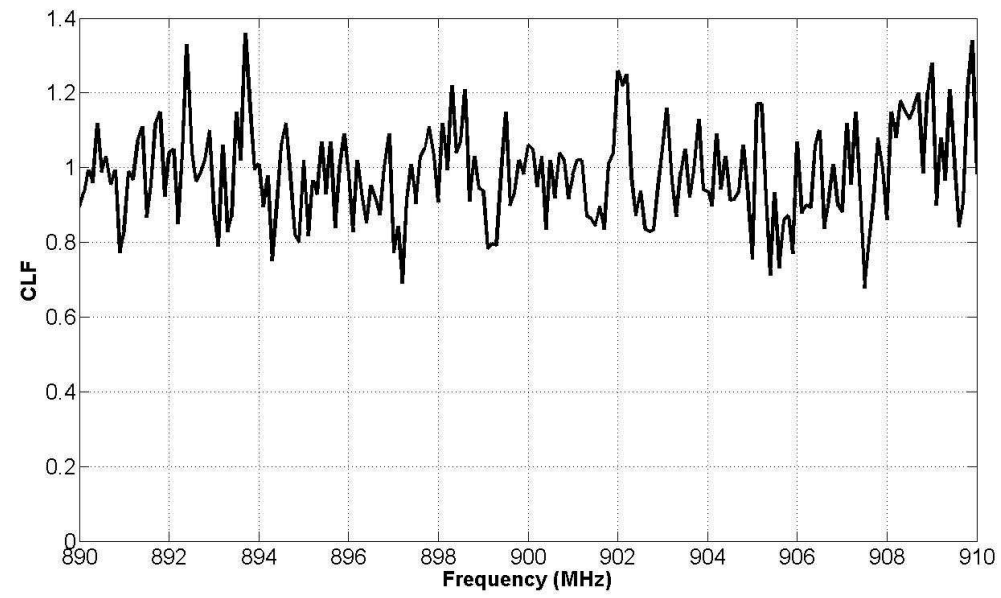

Figure 4. MSRC loading factor between $890 \mathrm{MHz}$ and $910 \mathrm{MHz}$.

Table 1. KS test for 3 points inside the MSRC working volume and outside the culture chamber, at $900 \mathrm{MHz}$.

\begin{tabular}{|c|c|c|c|c|}
\hline \multirow{2}{*}{$\begin{array}{c}\text { Measurements } \\
\text { locations }\end{array}$} & \multirow{2}{*}{$\begin{array}{c}\text { Measurements } \\
(\mathbf{9 0 0} \mathbf{M H z})\end{array}$} & \multicolumn{3}{|c|}{ Statistical KS levels } \\
\cline { 3 - 5 } & $\alpha=10 \%$ & $\alpha=5 \%$ & $\alpha=1 \%$ \\
\hline 1 & 0.0554 & 0.0993 & 0.1100 & 0.1320 \\
\hline 2 & 0.0313 & 0.1100 & 0.1220 & 0.1460 \\
\hline 3 & 0.0785 & 0.0997 & 0.1110 & 0.1330 \\
\hline
\end{tabular}

The loading factor, in the vicinity of $900 \mathrm{MHz}$, remains closed to 1 (Fig. 4).

In order to complete the analysis of the loaded MSRC, i.e., to verify the statistical parameters of the electromagnetic environment, we measured the power received by a log-periodic antenna for four points located inside the MSRC working volume and outside the incubator (stirring in continuous rotating mode). For independent samples extracted from these data, the KS statistical test was applied to the mean received power. As shown in Table 1, one noted nonrejection of the theoretical law of the power for a risk of first species equal to $1 \%, 5 \%$ and $10 \%$, this last value being the most constraining. Indeed, levels obtained for KS tests remained lower than the tabbed data (respectively for different $\alpha$ values). Consequently, the statistical properties of the electromagnetic fields in the working volume of the empty and loaded (by the incubator) MSRC appear similar (according 
to Fig. 3).

In order to verify the non influence of the electrical parts of the incubator, additional measurements of the electric field have been performed with and without these equipments in operation. The ratio between the averages (over a whole stirrer rotation) of the two series of the electric field values is lower than $1.5 \mathrm{~dB}$ (Fig. 5) that confirms the quasi independence of the electromagnetic field from the electrical devices (fan, heaters) in the incubator.

The last stage relating to the characterization of the electromagnetic field generated in the MSRC in the cells vicinity requires the measurement of the electric field inside the incubator.

Considering the field magnitude, the attenuation of an electric component due to the incubator structure was negligible as confirmed from Fig. 6. Indeed, means (for four measurement points) of the maximum value of the electric field component during each revolution of the stirrer [21] were similar $(7.87 \mathrm{~V} / \mathrm{m})$ within both the empty MSRC and within the incubator. Similarly to the previous case, considering [22], averaged value of total electric field remains steady with or without the biological incubator. Thus, the impact of the incubator on the electric field amplitude seems negligible, particularly at $900 \mathrm{MHz}$. The general restriction in MSRC obligates to place the biological dishes at a distance more than 0.5 wavelengths away from any metallic part and with a distance no less than 0.7 wavelengths from any absorber object. This last assumption was verified since

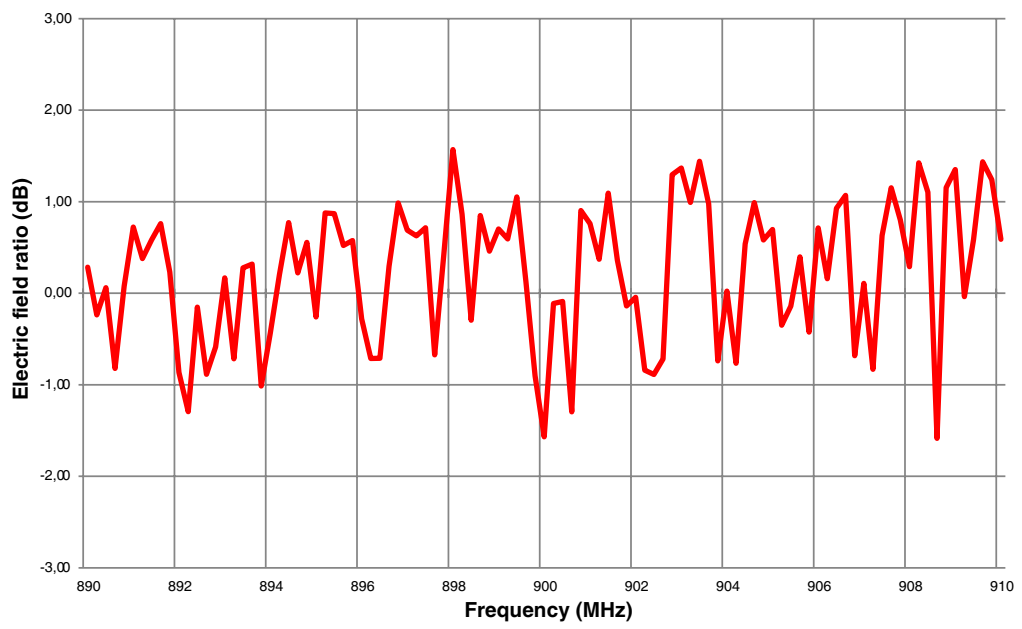

Figure 5. Ratio between the averages of the electric fields (inside the incubator) with and without electric devices in operation. 


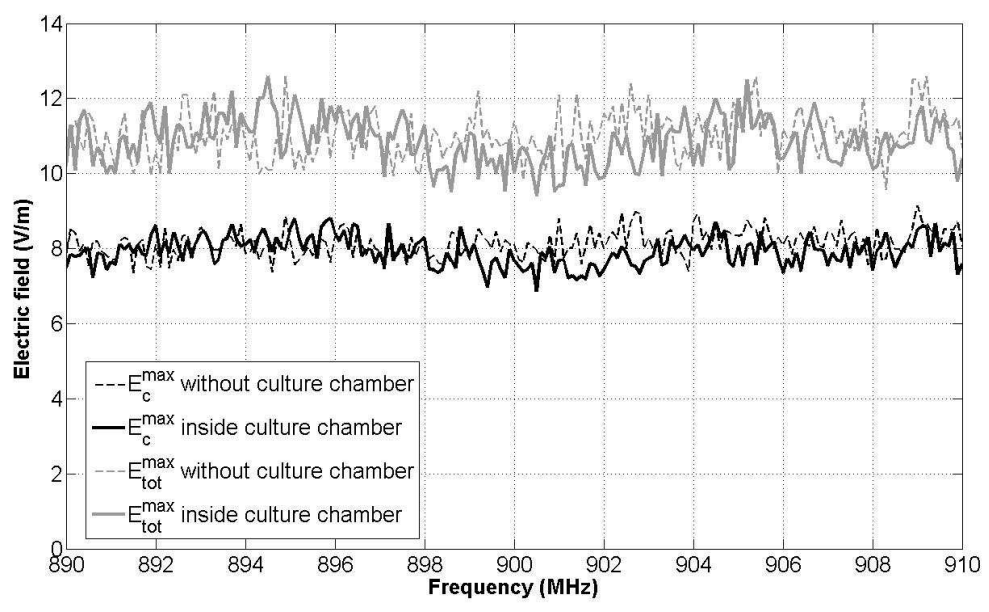

Figure 6. Electric fields with (solid) and without (dashed) culture chamber in MSRC. Measurements were treated according to $\stackrel{\leftrightarrow}{E_{c}}$ (black) and $\stackrel{\leftrightarrow}{E}_{\text {tot }}^{\max }$ (grey).

the incubator involves no major changing comparing inner and outer electric field (Fig. 6); it appeared transparent regarding to the electromagnetic environment. Moreover, from the description of the biological setup (Section 1.3), the heating devices and the air fan (Fig. 7) were located at a sufficient distance from living media (more or approximately $0.5 \lambda$, with $\lambda$ the wavelength for $f=900 \mathrm{MHz}$ ). Fig. 6 proved the relative electromagnetic transparency of the biological incubator (including heating devices, walls ...) since the differences between electric fields measured inside and outside the biological device were very slight. As expected, computing electric field from various criteria $\left(\stackrel{\leftrightarrow}{E_{c}}\right.$ and $\left.\stackrel{\leftrightarrow}{E_{\text {tot }}}\right)$ validated the good electromagnetic behavior of the incubator (i.e., transparency with regard to the HF EMF).

Further measurements of the electric field enabled to check right the electromagnetic behavior of the incubator inside the MSRC (following setup described on Fig. 7).

We also aimed to determine if the biological model is not exposed to very variable amplitudes of the field during a stirrer revolution $(9 \mathrm{~s})$. Thus, we measured the time evolution of the electric field around the biological model. From a statistical point of view, mean value of the total electric field (from the center of the incubator) is equal to $\bar{E}_{\text {tot }}^{\text {moy }}=5.85 \mathrm{~V} / \mathrm{m}$, with a standard deviation $(\mathrm{STD})$ of $0.769 \mathrm{~V} / \mathrm{m}$ in 
the neighborhood of cells (Fig. 8). Expressing dispersion of the electric field variations as the classical rate CV (Coefficient of Variation [36]) between standard deviation and mean value showed a relatively weak level (13\%). Moreover, about $60 \%$ of measured electric field levels remained between averaged value $(5.85 \mathrm{~V} / \mathrm{m}) \pm \mathrm{STD}$. Uniformity and isotropy of the inner electric field were checked: We first measured

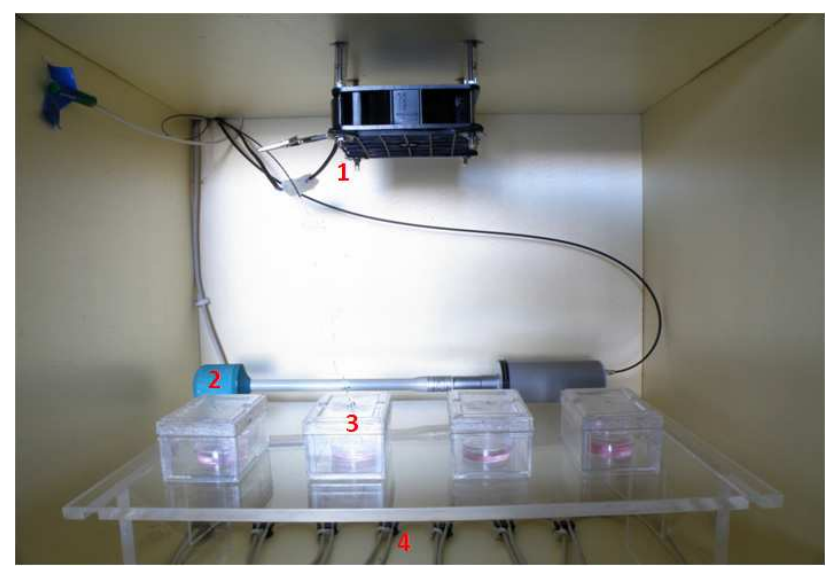

Figure 7. Experimental setup inside the culture chamber: air fan (1), electric field probe (2), individual cells cultures (3), heating device (4).

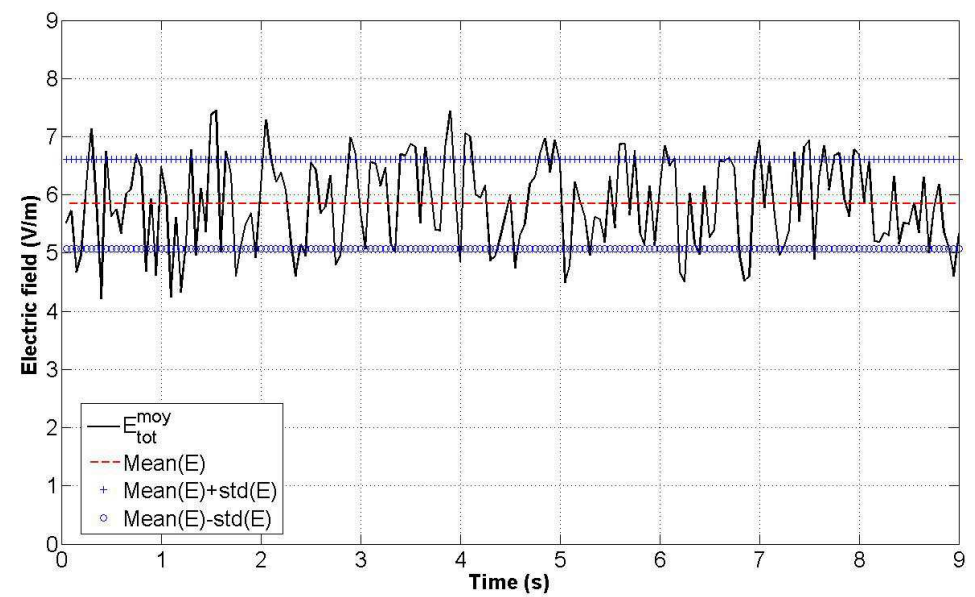

Figure 8. Time stability of the mean total electric field $\bar{E}_{\text {tot }}^{\text {moy }}$ over a stirrer rotation (180 steps). 
the magnitude of the three electric field components for four points inside the incubator, in tuning mode (i.e., for $N$ locations of the stirrer). Measuring the maximum value of each component during every revolution of the stirrer for all selected points led to compute standard deviations $\sigma_{x}, \sigma_{y}$ and $\sigma_{z}$ from each Cartesian component, and also $\sigma_{x y z}$ from the total electric field. Table 2 illustrates the properties of the HF EMF according to the [21] criteria. The standard deviations were determined from measurements at $900 \mathrm{MHz}$. As previously (empty case, Fig. 2), they were consistently lower than the $3 \mathrm{~dB}$ limit set by [21] specifications, verifying that the electromagnetic field in the vicinity of the biological cells was both homogeneous and isotropic. It could be noted that the measurements were performed in the vicinity of the plexiglass boxes but not inside them. Indeed, they were much too small to allow measurements inside them using the electric probe.

Then, we performed the measurement of the power received by a dipole antenna placed inside the incubator at the location of biological dishes at $900 \mathrm{MHz}$. Relying on the stirring mode operation, 500

Table 2. Standard deviations of the electric field (inside incubator, each component and total field).

\begin{tabular}{|c|c|c|c|c|}
\hline \multirow{2}{*}{ Frequency $\boldsymbol{f}$} & \multicolumn{4}{|c|}{ Standard deviations $\boldsymbol{\sigma}$ of internal electric field } \\
\cline { 2 - 5 } & $\sigma_{x}$ & $\sigma_{y}$ & $\sigma_{z}$ & $\sigma_{x y z}$ \\
\hline $900 \mathrm{MHz}$ & $1.37 \mathrm{~dB}$ & $1.22 \mathrm{~dB}$ & $0.68 \mathrm{~dB}$ & $1.07 \mathrm{~dB}$ \\
\hline
\end{tabular}

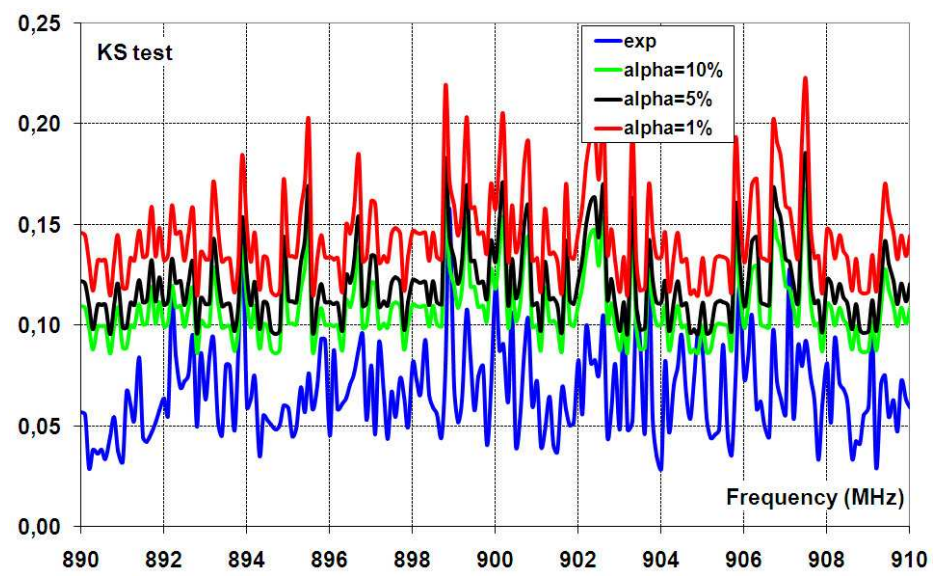

Figure 9. Results from statistical KS tests for measurements inside the culture chamber. 
measurements were obtained for one point in the vicinity of biological material. Fig. 9 shows the results concerning statistical KS tests to compare experimental (after extracting independent samples) and theoretical distributions.

The maximum gap existing between theoretical and experimental electric field distributions (named "exp" on Fig. 9) was lower than the limits set by [33] at $1 \%, 5 \%$ and $10 \%$ risks, respectively. It means that the probabilistic law of the electric field generated in the vicinity of the considered cells cultures was similar to the one obtained in the empty MSRC. We could therefore consider that biological dishes were irradiated by a statistically homogeneous and isotropic electric field. Consequently, the transparency of the thermo-regulated incubator was checked towards electromagnetic field properties around the biological samples.

The first part of this study highlighted the interest of using a MSRC as an exposure system for in vitro experiments. In contrast to a system generating a plane wave, the electromagnetic characteristics of the field are not statistically affected by the presence of the biological equipment. From Table 1, Fig. 3 and Fig. 9, the electromagnetic properties of power or field are checked respectively in the working volume outside from the incubator, in the empty MSRC and inside the biological device.

\section{THEORETICAL AND EXPERIMENTAL ANALYSIS OF THE SAR INSIDE THE BIOLOGICAL MEDIUM}

It was necessary to evaluate the homogeneity of the power density inside the biological medium where human cells grew. Comparing the small dimensions of the culture dishes to the MSRC size, the experimental determination of the SAR became extremely complex (from an electromagnetic point of view). We came to the conclusion that we had to develop a numerical model taking into account the whole electromagnetic reverberating environment. These results were then used to specify the experimental setup for SAR determination.

\subsection{SAR Computation with a FDTD Model Assuming Reverberating Environment}

From a physical point of view, dosimetry aimed to define the power absorbed by biological matter and media. Thus, the formula giving the expression of the SAR at $\mathbf{r}$ location inside the biological medium and at frequency $f$ was

$$
\operatorname{SAR}(\mathbf{r}, f)=\frac{\sigma}{\rho} E^{2}(\mathbf{r}, f)
$$


where $\sigma$ and $\rho$ stood respectively for the electric conductivity $(\mathrm{S} / \mathrm{m})$ and density $\left(\mathrm{kg} / \mathrm{m}^{3}\right)$ of biological medium. The internal electric field strength (root mean square) $E$ was measured in $\mathrm{V} / \mathrm{m}$. In the following, for simplicity, $\operatorname{SAR}(\mathbf{r}, f)$ might be written $\operatorname{SAR}(f)$ referring to a single numerical location $\mathbf{r}$.

Based upon previous relation (7), SAR simulations needed to compute the most accurate value of the electric field $E$. From the variety of methods (frequency and time domains for instance), we chose the Finite-Difference in Time-Domain (FDTD) technique [34]. Considering the constant increase of computing power, the advantages of this method were numerous: simplicity, robustness, analysis on a large frequency bandwidth. The method allowed to model various sources, free-space and different materials (dispersive, dissipative, non linear ones). Based on these arguments, this formalism has already been successfully used to compute dosimetric quantities [35] (typical dielectric needs due to the presence of living materials).

Considering the large spread of FDTD theoretical principles, this section is dedicated to a brief summing up of necessary elements to understand the basis of SAR computing achieved in this study. FDTD basically relied on writing the Maxwell's equations in a differential form, then simplifying those different space and time derivatives by a technique called "Finite-Difference". The method therefore required a space discretization of the computational domain following Yee's cell (Fig. 10(a)).

We were interested in SAR computing inside biological medium; this simulated value stood for the power density absorbed in each culture dish. It might be noticed, from various studies [35], that a sharp electromagnetic dosimetric analysis (around millimeters for a frequency of $900 \mathrm{MHz}$ ) was required to define SAR levels inside biological media. Obviously, modeling the complete system including the cavity, the mechanical rotating mode stirrer, the biological incubator and the dishes, involved numerical difficulties (huge memory and time computing costs). Nevertheless, simulations allowed to describe more precisely the power density distribution (uniform or not) for each biological dishes. As explained in the following, FDTD models (Fig. 10(b)) enabled to combine accurate description of biological media (including the culture liquid with the electromagnetic characteristics $\epsilon_{r}=71$ and $\sigma=2 \mathrm{~S} / \mathrm{m}$, and plexiglass dishes surrounding it, $\mathrm{CO}_{2}$ biological container were not included) and an attractive description of the inner electromagnetic environment inside the MSRC.

From a numerical point of view, the huge scaling factor existing between sizes of the electromagnetic device (MSRC, volume $\cong 200 \mathrm{~m}^{3}$ ) 


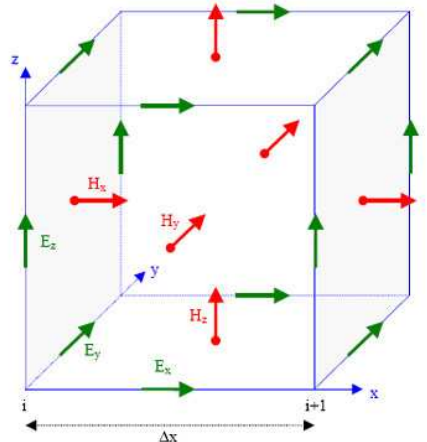

(a)

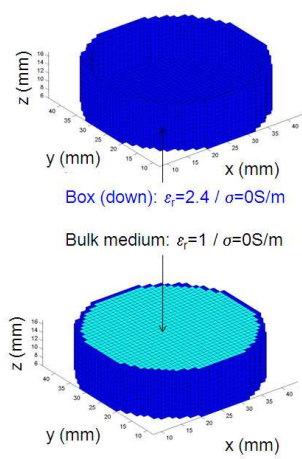

(b)
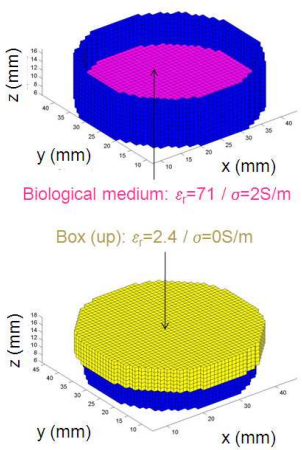

Figure 10. (a) Yee's cell. (b) FDTD models for biological dishes.

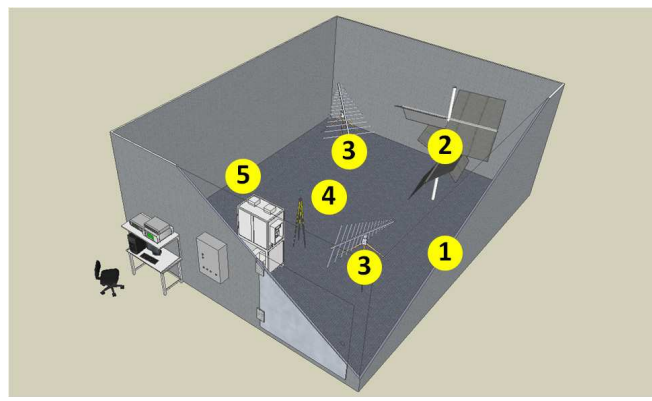

(a)

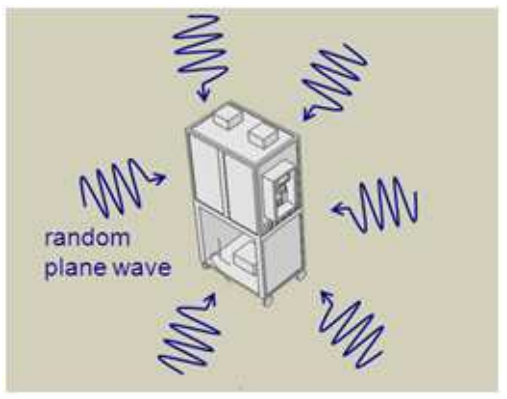

(b)

Figure 11. (a) MSRC setup including walls (1), stirrer (2), antennas (3), field probe (4), biological incubator under test (5). (b) Finite sum of plane waves (similar to MSRC behavior).

and the irradiated element (culture dishes, volume of biological medium $=2 \mathrm{~mL}$ ) required a particular numerical strategy. Relying on previous theoretical works describing a plane wave modeling of the electric field inside the MSRC, the electromagnetic reverberating environment might be numerically approached by a finite sum of plane waves [14].

Figure 11 gives a sketch of the previous analogy applied on the biological incubator. From a numerical point of view, the measurement device used for classical MSRC tests needs a whole description of experimental equipments: The entire volume of the metallic cavity, 
the mechanical stirrer and antennas must be modeled. Generally, the complexity of MSRC simulations is given as a function of the volume of the enclosure (i.e., the number of unknowns), the losses observed inside the culture chamber, the frequency bandwidth of study and the intrinsic characteristics (physical and geometrical properties) of the equipment. Considering SAR expectations of these works and previous remarks about sizes of the MSRC, the different scales of equipments prevent to straightforward modelling the MSRC and the biological medium (prohibitive time and memory costs). Consequently we proposed a randomly distributed plane wave approach gathered with FDTD numerical tools [34].

The method described in this study was applied successfully to simulate the exposure of animals in a reverberating environment in [14]. The method basically involved the illumination of the biological model with a finite (twelve) number of plane waves with the same magnitude but with different polarizations and incident directions. The total SAR was numerically assessed in each voxel (FDTD elementary cell) as a superposition of magnitudes (SAR) and normalized according to the empty setup.

One of the main advantages of time techniques relied on their ability to work on a large frequency bandwidth in a single simulation. Results given by [35] fitted with those obtained from single numerical irradiation (one plane wave impinging on biological dish in our study). This last remark laid emphasis on the importance of normalization with power (or electric field) inserted. From SAR relation (7) and previous plane wave description, the standardized SAR simulation in a reverberating environment (unitary mean magnitude) for each frequency $f$ was given by

$$
\operatorname{NSAR}_{(1 \mathrm{~V} / \mathrm{m})}(f)=\frac{\operatorname{SAR}(f)}{\left\langle E_{e}^{2}(f)\right\rangle}
$$

where $\left\langle E_{e}^{2}(f)\right\rangle$ stood for the mean squared electric field without biological medium (i.e., averaged value of $E^{2}$ inside simulated working volume in empty case). $\operatorname{NSAR}_{(1 \mathrm{~V} / \mathrm{m})}(f)$ was given in $\mathrm{W} \cdot \mathrm{m}^{2} /\left(\mathrm{kg} \cdot \mathrm{V}^{2}\right)$ and represented a normalized SAR value at frequency $f$.

Consequently, regarding the numerical exposure field distribution as a realistic representation of the genuine electric field distribution inside the MSRC [14], we could gather simulated and measured SAR. Indeed, taking into account the incident power (numerically or experimentally), the mean value $x$ of the electric field observed in reverberating environment might be integrated in final SAR computation as follows:

$$
\operatorname{SAR}_{(x \mathrm{~V} / \mathrm{m})}(f)=x^{2} \cdot \mathrm{NSAR}_{(1 \mathrm{~V} / \mathrm{m})}(f)
$$




\subsection{Simulated SAR and Discussion}

As explained previously, an important point of the characterization of the electromagnetic configuration concerned the SAR determination. We estimated its spatial distribution by electromagnetic simulations based on FDTD in order to access further analysis concerning SAR computation. The FDTD model described on Fig. 12(b) represented one biological dish that was irradiated by a random sum of plane waves (obviously, numerically, a finite one, 198 similarly to [15]). Based upon this previous reference (200 plane waves), the discretization (FDTD) of the numerical setup imposed criteria (number) to properly model the plane waves description (incidence uniformly distributed). To carefully describe the plane waves distribution, the closer number plane waves regarding [15] was 198. Physical parameters given on Fig. 10(b) (relative electric permittivity $\epsilon_{r}$ and conductivity $\sigma$ for $f=900 \mathrm{MHz}$ ) enabled to compute accurately SAR inside $2 \mathrm{~mL}$ of culture medium relying on relation (7): $12 \mathrm{~mm}$ height and $41 \mathrm{~mm}$ cylindrical shape.

Figure 12 gives an overview of the SAR distributions in linear scale considering minimum and maximum values obtained respectively for a polarized (one plane wave illuminating top of dish) and random source at $900 \mathrm{MHz}$. The magnitude of the total electric field used on Fig. 12 was fixed $\bar{E}_{t o t}^{\text {moy }}=5.85 \mathrm{~V} / \mathrm{m}$. Distributions relied on properties of electromagnetic sources (results from Figs. 12(a) and 12(b) laid emphasis on the importance of polarization, incidence and number of plane waves numerically used to fit with MSRC environment).

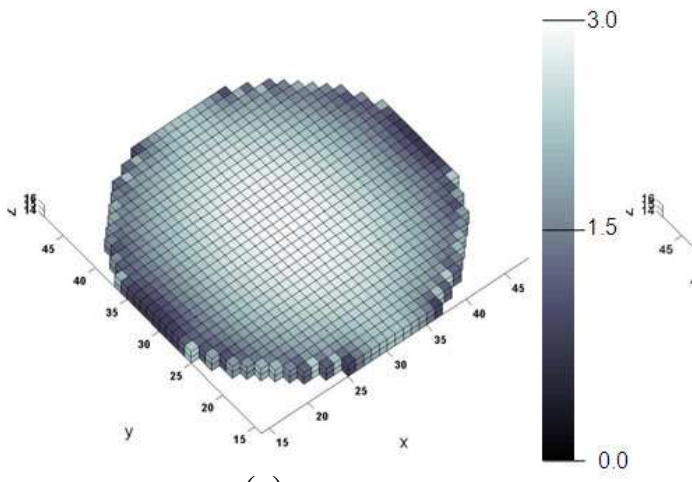

(a)

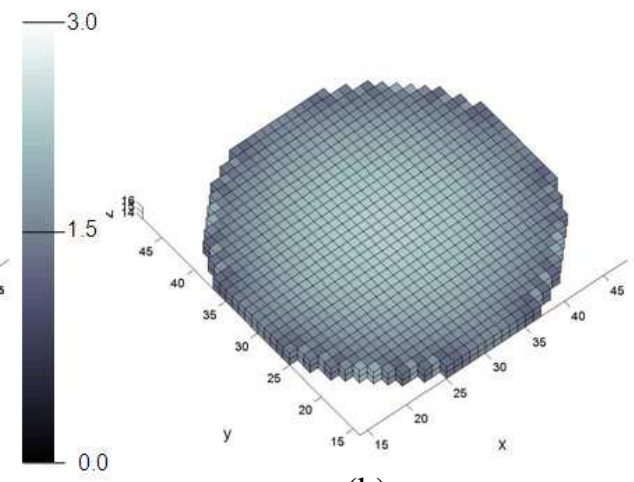

(b)

Figure 12. SAR distribution (in $\mathrm{mW} / \mathrm{kg}, f=900 \mathrm{MHz}$, ambient electric field $\bar{E}_{\text {tot }}^{\text {moy }}=5.85 \mathrm{~V} / \mathrm{m}$ ) inside one dish for 1 polarized source (a) and randomly distributed/polarized source of 198 plane waves (b). 
Table 3. Statistical data from normalized SAR for one culture dish and two levels of stimulation.

\begin{tabular}{|c|c|c|c|c|}
\hline \multirow{2}{*}{$\begin{array}{c}\text { Level (SAR } \\
\text { normalization) }\end{array}$} & \multicolumn{4}{|c|}{ SAR (statistical data) in $\mathbf{m W} / \mathbf{k g}$} \\
\cline { 2 - 5 } & $\langle\mathrm{SAR}\rangle$ & $\min _{\mathrm{SAR}}$ & $\max _{\mathrm{SAR}}$ & $\sigma_{\mathrm{SAR}}(\mathrm{STD})$ \\
\hline Level 1 & 2.0 & 1.3 & 2.5 & 0.3 \\
\hline Level 2 & 54 & 35 & 68 & 8.1 \\
\hline
\end{tabular}

Results given on Fig. 12(a) were in a good agreement with conclusions from [35] regarding the importance of polarization for in vitro electromagnetic exposure. SAR was not homogeneous inside biological dishes and relatively huge SAR gradients appeared (i.e., cells in culture did not receive the same power during experiment). In this context, the polarization of irradiating plane wave has an important impact both on SAR strengths (from $0.7 \mathrm{~mW} / \mathrm{kg}$ to $3.2 \mathrm{~mW} / \mathrm{kg}$ ) and on its distribution. In contrast to the previous case, for a randomly distributed/polarized source, the three-dimensional SAR distribution might be considered as homogeneous inside the culture medium, i.e., the individual repartition of each SAR FDTD cell in biological meshing was approximately uniform (Fig. 12(b)). Statistical SAR data were summed up in Table 3 (level 1) and laid emphasis on relatively weak SAR variations around the mean value $(2 \mathrm{~mW} / \mathrm{kg})$. Even if heat points appeared on the interface between the culture medium and the plexiglass dish (on the neighbourhood in Fig. 12(b)), the SAR gradients were much weaker than for the polarized source (Fig. 12(a)).

Previous works [35] focused on the difficulty to fit accurate SAR computation with fine description of devices used for EMC tests. Thus, experiments performed in the MSRC allowed to take advantage of a large number of electromagnetic excitations (various angles of incidence and polarization for a statistically uniform electric field). Consequently, the biological device was irradiated with the most penalizing configuration.

SAR distributions respectively for polarized/random sources (overview was given on Fig. 12) were detailed in the histograms on Fig. 13. SAR simulated values (linear scale) were organized according to 15 classes and represented considering the relative frequency of the $\mathrm{SAR}$ value in each case. In a first time, regarding Fig. 13(a) (one polarized plane wave source), the total average SAR was $2.3 \mathrm{~mW} / \mathrm{kg}$; $45.3 \%$ of cells had SAR values between $1.71 \mathrm{~mW} / \mathrm{kg}$ and $2.35 \mathrm{~mW} / \mathrm{kg}$; $14.1 \%$ of cells had SAR values lower than $1.71 \mathrm{~mW} / \mathrm{kg} ; 40.6 \%$ of cells had SAR values higher than $2.35 \mathrm{~mW} / \mathrm{kg}$. In a second time, considering Fig. 13(b) (randomly distributed/polarized plane wave source), the total average SAR was $2.0 \mathrm{~mW} / \mathrm{kg} ; 87.6 \%$ of cells had SAR values 
between $1.71 \mathrm{~mW} / \mathrm{kg}$ and $2.35 \mathrm{~mW} / \mathrm{kg} ; 7.8 \%$ of cells had SAR values lower than $1.71 \mathrm{~mW} / \mathrm{kg} ; 4.6 \%$ of cells had SAR values higher than $2.35 \mathrm{~mW} / \mathrm{kg}$.

From previous results, the three-dimensional SAR distribution was relatively homogeneous for a random source compared to the polarized case since most of the SAR values were close to the average (Fig. 13(b)) contrary to the polarised exposure [35]. Indeed, SAR maxima (Fig. 13(a)) were concentrated in the last class justifying a worse SAR distribution regarding the homogeneity. Finally, those numerical

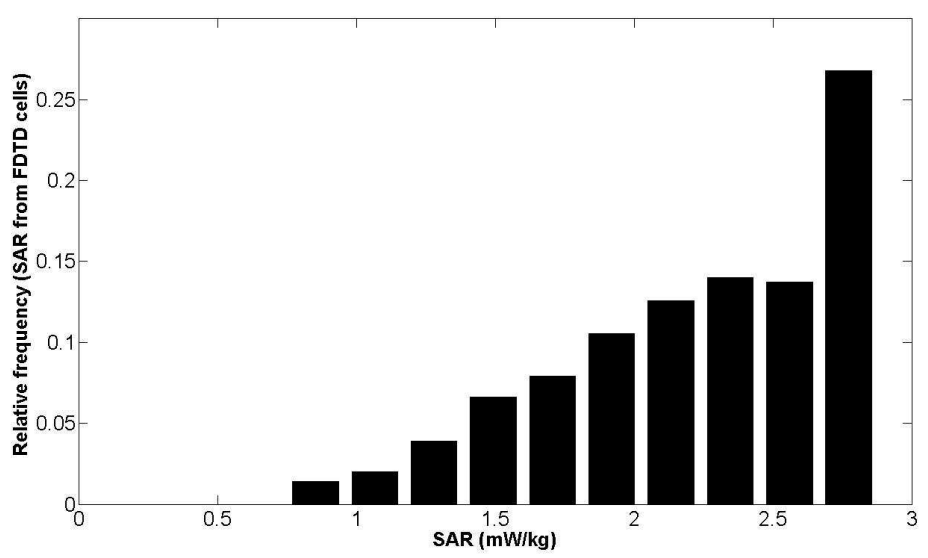

(a)

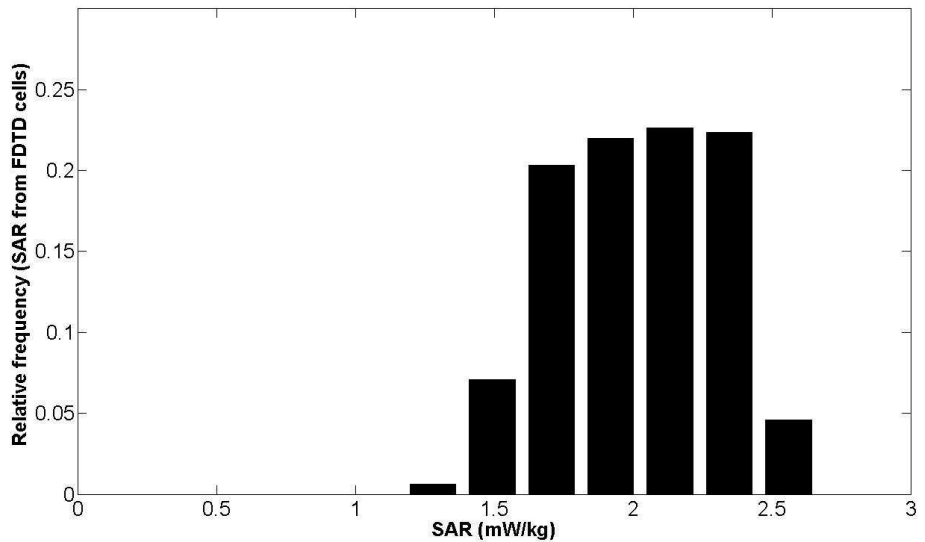

(b)

Figure 13. Relative frequency of local SAR values (in $\mathrm{mW} / \mathrm{kg}$, $f=900 \mathrm{MHz}$, ambient electric field $\left.\bar{E}_{\text {tot }}^{\text {moy }}=5.85 \mathrm{~V} / \mathrm{m}\right)$ for polarized source (a) and random source of 198 plane waves (b). 
results obtained at $900 \mathrm{MHz}$ justified the use of a single central temperature value in the biological medium for SAR determination in the MSRC.

Computed values were normalized for two different levels of stimulation to allow precise comparison of SAR simulations. Levels 1 and 2 stood for mean total electric field $\bar{E}_{\text {tot }}^{\text {moy }}=5.85 \mathrm{~V} / \mathrm{m}$ and $30.47 \mathrm{~V} / \mathrm{m}$ (respectively for $\stackrel{\leftrightarrow}{E_{\text {tot }}}=7.87 \mathrm{~V} / \mathrm{m}$ and $41 \mathrm{~V} / \mathrm{m}$ ). In the following, both results given by numerical and measured experiments were normalized by levels 1 and 2. Table 3 sum up the results obtained from stimulation of one biological dish by a random sum of plane waves. Mean SAR values were about $2.0 \mathrm{~mW} / \mathrm{kg}$ and $54 \mathrm{~mW} / \mathrm{kg}$ respectively for levels 1 and 2 .

We were interested to more precisely understand the SAR measurements with results taken from computation. Obviously, effects of coupling between various biological dishes were rather complex to study experimentally. Considering the experimental setup (number of dishes, distance between them), further simulations were achieved inserting several biological devices (including culture medium and dish) in order to rate the importance of inter-dish coupling.

Although some coupling effects may appear [35] considering one polarized plane wave, only low levels of coupling were observed in this study. The relative gap (CE) existing between each dish (considered independently) and the "1 dish" simulation enabled to easily compute the coupling effects.

Technically, comparing "1 dish" with "4 dishes" simulations to evaluate coupling effects meant computing, for each FDTD cell $i$, the relative gap between the reference electromagnetic field ("1 dish", no coupling, $\left.E_{i}^{0}\right)$ and the one obtained in the presence of 4 dishes $\left(E_{i}^{j}\right.$, $j=$ number of dish, Fig. 14)

$$
C E_{i}^{j}=\left|\frac{E_{i}^{0}-E_{i}^{j}}{E_{i}^{0}}\right|
$$

Obviously, a relative gap $C E_{i}^{j}=0$ stood for no coupling effect in FDTD cell $i$ of dish $j$.

Thus, considering Table 4, a closer look was given to the impact of coupling for FDTD simulation involving 198 random plane waves (with uniform distribution of incidence and polarization). Although some effects appeared with multiple dishes, the results reported in Table 4 underlines relatively weak levels of coupling (maximum 5\% of coupling effect). Accordingly, no major coupling was expected in SAR measurements in the MSRC. 
Table 4. Coupling effects (" 4 dishes" compared to " 1 dish" case).

\begin{tabular}{|c|c|c|c|c|}
\hline & \multicolumn{4}{|c|}{$\begin{array}{c}\text { Coupling effects (CE) for a FDTD } \\
\text { source composed of 198 random } \\
\text { plane waves (\% gap for each dish) }\end{array}$} \\
\hline $\begin{array}{c}\text { Biological dish number } \\
\text { (Fig. 14) }\end{array}$ & 1 & 2 & 3 & 4 \\
\hline CE (10) mean (over all FDTD cells) & $1.15 \%$ & $1.42 \%$ & $1.40 \%$ & $1.98 \%$ \\
\hline CE (10) minimum & $0.002 \%$ & $0.005 \%$ & $0.007 \%$ & $0.001 \%$ \\
\hline CE (10) maximum & $3.68 \%$ & $4.05 \%$ & $3.91 \%$ & $5.03 \%$ \\
\hline
\end{tabular}

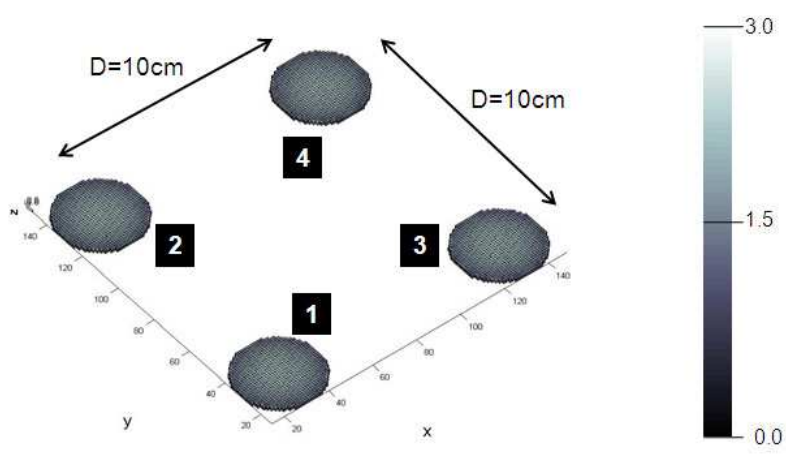

Figure 14. SAR distribution (in $\mathrm{mW} / \mathrm{kg}, f=900 \mathrm{MHz}$, ambient electric field $5.85 \mathrm{~V} / \mathrm{m}$ ) of four cells culture dishes irradiated by random source (198 randomly distributed/polarized plane waves).

The use of a numerical model appeared essential to the analysis of SAR homogeneity in the biological medium. Moreover accessing to the actual value of SAR revealed very interesting in order to guarantee dosimetric levels expected for MSRC experiments. Numerical results were normalized with the level of field generated experimentally (levels 1 and 2). Results from a "4 dishes FDTD simulation" are reported on Fig. 14 using the same source of random plane waves (Fig. 12(b)). The presence of four dishes instead of one did not seem to cause major changes in SAR distribution inside each plate (Fig. 14).

From a purely numerical point of view, the simulations of the whole experiments (including FDTD models and random sources) were achieved on a 'desktop' personal computer (processor Intel Quad $2.40 \mathrm{GHz} / 3.25$ Go RAM). For "1 dish" and "4 dishes" tests, they needed a CPU time of about 1.5 days and 10.5 days. Finally, the requested memories were respectively 20 Mo and 184 Mo. 


\subsection{SAR Validation from Experiments}

\subsubsection{SAR from Temperature Measurements}

The numerical SAR values obtained from a FDTD code were faced to experimental data from temperature measurements. It was widely admitted that SAR evaluation (in $\mathrm{W} / \mathrm{kg}$ ) might be obtained from

$$
\mathrm{SAR}=\left.C \frac{d T}{d t}\right|_{t=0}
$$

where $C$ stands for the biological medium heat capacity in $\mathrm{J} /\left(\mathrm{kg} \cdot{ }^{\circ} \mathrm{C}\right)$, $T$ represents temperature in ${ }^{\circ} \mathrm{C}$, and $t$ (in s) is time of exposure. From relation (11), one can note that the time derivative is necessary at the initial instant. That is the reason why numerical treatments are needed to fit the temperature curve at the origin of the electromagnetic exposure. In the following, one of the major issues was to properly define a setup allowing determination of the initial time of exposure (see following section regarding experimental dosimetry). Thus a linear fitting enabled to compute the time derivative in (11) from temperature measurements. Moreover, results given by numerical SAR simulations justified the choices concerning SAR experimental setup.

\subsubsection{Experimental Dosimetry}

According to the relation (11), SAR might be obtained from temperature measurements (numerical or experimental ones). In this study, this way was used to evaluate SAR experimentally and to compare those results with numerical data from previous section. From numerical dosimetric study, it was established that measurement may be achieved for one location and one dish (at the centre of one biological dish), relying on computed SAR distributions and low levels of coupling between biological dishes. Temperature measurements were done using a Luxtron probe (temperature monitoring Luxtron 812 and STF Luxtron probe, Fig. 15).

It is worth noting that no temperature increasing was recorded for the two levels of exposure previously defined (levels 1 and 2, respectively, $\stackrel{\leftrightarrow}{E_{c}}=7.87 \mathrm{~V} / \mathrm{m}$ and $\left.41 \mathrm{~V} / \mathrm{m}\right)$. Complementary SAR experiments were led by increasing levels of electric fields in order to access measurable temperature variations. The mean value of SAR over a whole rotation of the stirrer was obtained from three series of data corresponding to three different levels of power transmitted to the MSRC (called cases A, B and C in Tables 5 and 6).

Temperature measurements were done as described by [37]: After checking the temperature stability, a sufficient amount of power was 


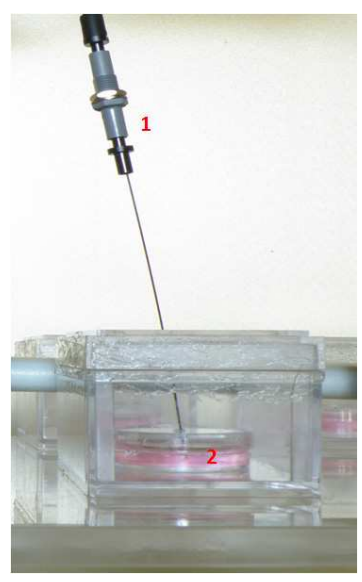

Figure 15. Temperature measurements: LUXTRON STF probe 1, individual biological enclosure 2 with central measurement location.

Table 5. Powers inserted inside MSRC and corresponding electric fields (SAR measurements).

\begin{tabular}{|c|c|c|c|}
\hline & Case A & Case B & Case C \\
\hline$P_{\text {in }}(\mathrm{W})$ & 78.16 & 63.39 & 33.50 \\
\hline$\stackrel{\leftrightarrow}{E}_{c}(\mathrm{~V} / \mathrm{m})$ & 343 & 309 & 224 \\
\hline $\bar{E}_{\text {tot }}^{\text {moy }}(\mathrm{V} / \mathrm{m})$ & 255 & 230 & 167 \\
\hline
\end{tabular}

injected to allow a recordable temperature increase inside the biological samples (Fig. 16, using Luxtron probe referred in Fig. 15 label).

Table 5 defines the levels of the incident power $P_{\text {in }}$ inside the MSRC. For these three levels, the magnitude of the electric field $\stackrel{\leftrightarrow}{E}_{c}$ was measured according to (4) and [21]. The averaged magnitude of the total electric field $\bar{E}_{\text {tot }}^{\text {moy }}$ (quantity more representative for dosimetric experiments) was also computed to enable an accurate comparison with simulated data.

The SAR was determined by performing measurements every $0.5 \mathrm{~s}$. Given the fact that one rotation of the stirrer lasted $9 \mathrm{~s}, 200 \mathrm{~s}$ (400 measurements) were accounting for more than 20 revolutions available. The experimental SAR was deduced (linear fitting) from temperature (Fig. 16). Three different series of measurements were realized, allowing testing the repeatability of the SAR protocol.

We recorded the time profiles of temperatures for each value of injected power in order to evaluate levels of SAR inside the human 


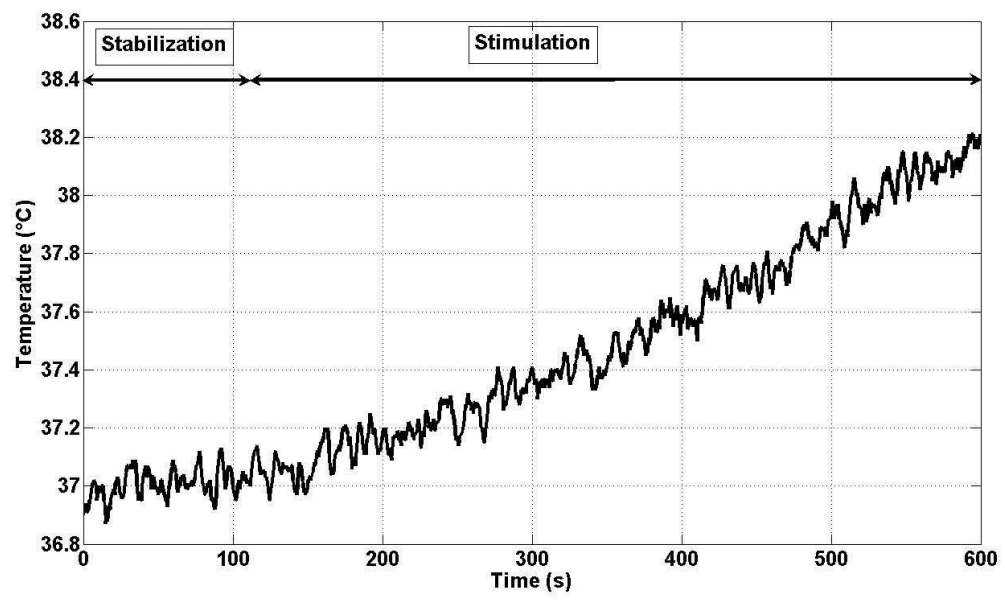

Figure 16. Temperature measurements with Luxtron apparatus (power inserted from case C, Table 5).

Table 6. SAR measurements $(\mathrm{mW} / \mathrm{kg})$ for two normalized exposure levels (3 setups: cases $\mathrm{A}, \mathrm{B}$ and $\mathrm{C}$ ).

\begin{tabular}{|c|c|c|c|c|c|}
\hline \multirow{2}{*}{ Stimulation } & \multicolumn{5}{|c|}{ SAR $\mathbf{( m W} / \mathbf{k g})$} \\
\cline { 2 - 6 } & Case A & Case B & Case C & $\langle\mathrm{SAR}\rangle$ & $\sigma_{\text {SAR }}(\mathrm{STD})$ \\
\hline Level 1 & 2.3 & 3.0 & 2.8 & 2.6 & 0.4 \\
\hline Level 2 & 61 & 81 & 75 & 73 & 9.9 \\
\hline
\end{tabular}

cells culture medium. Then, these values were respectively normalized with levels 1 and 2 of electric field $\left(\bar{E}_{\text {tot }}^{\text {moy }}=5.85 \mathrm{~V} / \mathrm{m}\right.$ and $30.47 \mathrm{~V} / \mathrm{m}$ corresponding to the average of total electric field inside the culture dish over a complete rotation of the stirrer). Normalization of SAR results according to previous levels of stimulation for the various setups (cases A, B and C) is given (Table 5).

From values given by Table 6 , the weak scattering of results from cases $\mathrm{A}, \mathrm{B}$ and $\mathrm{C}$ around mean value (average $\langle\mathrm{SAR}\rangle$ ) might be noted. Thus, because of the validation of the experiments repeatability for various values of inserted power, we deduced SAR levels for bioelectromagnetic exposures achieved. The mean value of SAR for each revolution was respectively equal to $2.6 \mathrm{~mW} / \mathrm{kg}$ and $73 \mathrm{~mW} / \mathrm{kg}$ for levels 1 and 2 .

Finally, experimental results were in a good agreement with numerical data (Table 3: Peak values respectively of $2.5 \mathrm{~mW} / \mathrm{kg}$ and 
$68 \mathrm{~mW} / \mathrm{kg}$ were obtained inside biological medium for exposure levels 1 and 2, and central location). Moreover, this analysis showed the complementarity between experimental and theoretical approaches to characterize the exposure system.

\section{CONCLUSIONS AND PROSPECTS}

This article showed that MSRC is an efficient electromagnetic device for in vitro bioelectromagnetism tests. Relying on its ability to control characteristics of electromagnetic environment in a given working volume, many benefits might be expected for bioelectroMagnetic Compatibility (bioEMC) experiments. From a statistical point of view, distribution laws of electric field were obtained considering data during a whole revolution of the stirrer. The field properties were accurately known in both empty and loaded chamber. The physical properties of the biological material (culture medium, dishes and incubator) has no influence on electromagnetic distribution. The quality of the environment in the vicinity of human cells in culture was given by the study of statistical electromagnetic properties obtained from measurements of electric field inside the incubator. Then, particular works were achieved to compute SAR inside the culture medium. Experimental and numerical experiments both showed that low SAR levels (respectively $2.6 \mathrm{~mW} / \mathrm{kg}$ and $2.0 \mathrm{~mW} / \mathrm{kg}$ ) were obtained in the MSRC with an inner electric field $\bar{E}_{\text {tot }}^{\text {moy }}=5.85 \mathrm{~V} / \mathrm{m}$. Moreover SAR distribution revealed relatively uniform inside biological dishes. Contrary to temperature measurements, SAR modeling relied on computing electromagnetic distributions and allowed analysis of coupling effects between culture media: Numerical results given for four biological dishes showed no major effect of mutual coupling between them. Moreover, they justified the use of a single location for SAR experiments. The experimental approach gave references to evaluate two SAR results corresponding to biological experiments achieved respectively with low and high amplitudes of irradiation. Obviously, both the exposure system and the levels of incident power may be taken into account to properly quantify SAR values. Finally, the use of MSRC for bioEMC appeared as a convenient facility to achieve frontiers between physical and biological works. Experience earned from EMC studies in MSRC has been adapted and improved considering current electromagnetic works about biological irradiation and SAR definitions. 


\section{REFERENCES}

1. Burkhardt, M., K. Pokovic, M. Gnos, T. Schmid, and N. Kuster, "Numerical and experimental dosimetry of Petri dish exposure setups," Bioelectromagnetics, Vol. 17, 483-493, 1996.

2. Paffi, A., R. Pinto, M. Liberti, F. Apollonio, G. A. Lovisolo, and G. D'Inzeo, "Review of exposure setups for biological experiments in the radiofrequency range specifications and emerging trends," URSI General Assembly, 2008.

3. Nikoloski, N., J. Frohlich, T. Samaras, J. Schuderer, and N. Kuster, "Reevaluation and improved design of the TEM cell in vitro exposure unit for replication studies," Bioelectromagnetics, Vol. 26, 215-224, 2005.

4. Duranti, G., A. Rossi, N. Rosato, and G. Fazio, "In vitro evaluation of biological effects on human keratinocytes exposed to $900 \mathrm{MHz}$ electromagnetic field," The Environmentalist, Vol. 25, 113-119, 2005.

5. Jorge-Mora, T., M. Alvarez-Folgueiras, J. Leiro, F. J. JorgeBarreiro, F. J. Ares-Pena, and E. Lopez-Martin, "Exposure to $2.45 \mathrm{GHz}$ microwave radiation provokes cerebral changes in induction of HSP-90/heat shock protein in rat," Progress In Electromagnetics Research, Vol. 100, 351-379, 2010.

6. Penafiel, L. M., T. Litovitz, D. Krause, A. Desta, and J. M. Mullins, "Role of modulation on the effect of microwaves on ornithine decarboxilase activity in L929 cell," Bioelectromagnetics, Vol. 18, 132-141, 1997.

7. Laval, L., P. Lévêque, and B. Jecko, "A new in vitro exposure device for the mobile frequency of $900 \mathrm{MHz}, "$ Bioelectromagnetics, Vol. 20, 1-9, 1999.

8. De Gannes, F. P., M. Taxile, S. Duleu, A. Hurtier, E. Haro, M. Geffard, G. Ruffié, B. Billaudel, P. Lévêque, P. Dufour, I. Lagroye, and B. Veyret, "A confirmation study of russian and ukrainian data on effects of $2450 \mathrm{MHz}$ microwave exposure on immunological processes and teratology in rats," Radiation Research, Vol. 172, No. 5, 617-624, November 2009.

9. Iyama, T., H. Ebara, Y. Tarusawa, S. Uebayashi, M. Sekijima, T. Nojima, and J. Miyakoshi, "Large scale in vitro experiment system for $2 \mathrm{GHz}$ exposure," Bioelectromagnetics, Vol. 25, 599606, 2004.

10. International Commission on Non-ionizing Radiation Protection, "Guidelines for limiting exposure to time-varying electric, magnetic, and electromagnetic fields (up to $300 \mathrm{GHz}$ )," Health 
Physics, Vol. 74, No. 4, 494-522, 1998.

11. Sharma, V., H. Singh, R. Kohli, and D. Batish, "Mobile phone radiation inhibits vigna radiata (mung bean) root growth by inducing oxidative stress," Science of the Total Environment, Vol. 407, 5543-5547, 2009.

12. Ladbury, J., P. Wilson, G. Koepke, and T. Lammers, "Reverberation chamber: An evaluation for possible use as a RF exposure system for animal studies," Bioelectromagnetics Soc. (BEMS) 25th Annu. Meeting, 150-151, Maui, HI, June 22-27, 2003.

13. Biagi, P. F., L. Castellana, T. Maggipinto, G. Maggipinto, T. Ligonzo, L. Schiavulli, and D. Loiacono, "A reverberation chamber to investigate the possible effects of 'in vivo' exposure of rats to $1.8 \mathrm{GHz}$ electromagnetic fields: A preliminary study," Progress In Electromagnetics Research, Vol. 94, 133-152, 2009.

14. Berdinas-Torres, V. J., "Exposure's systems and dosimetry of large-scale in vivo studies," Doctoral Thesis, Swiss Federal Institute of Technology, Zurich, 2007.

15. Wu, T., A. Hadjem, M. F. Wong, A. Gati, O. Picon, and J. Wiart, "Whole-body new-born and yound rats' exposure assessment in a reverberating chamber operating at $2.4 \mathrm{GHz}$," Physics in Medicine and Biology, Vol. 55, 1619-1630, 2010.

16. "Mise à jour de l'expertise relative aux radiofréquences," Rapport d'expertise Collective Afsset, Groupe de Travail Radiofréquences, Saisine, n2007/007, October 2009.

17. Beaubois, E., S. Girard, S. Lalléchère, E. Davies, F. Paladian, P. Bonnet, G. Ledoigt, and A. Vian, "Intercellular communication in plants: Evidence for two rapidly systemic signals generated in response to electromagnetic field stimulation in tomato," Plant Cell and Environment, Vol. 30, 834-844, 2007.

18. Vian, A., D. Roux, S. Girard, P. Bonnet, F. Paladian, E. Davies, and G. Ledoigt, "Microwave irradiation affects gene expression in plants," Plant Signaling and Behavior, Vol. 1, No. 2, 67-69, 2006.

19. Roux, D., A. Vian, S. Girard, P. Bonnet, F. Paladian, E. Davies, and G. Ledoigt, "Electromagnetic fields $(900 \mathrm{MHz})$ evoke consistent molecular responses in tomato plants," Phys. Plant, Vol. 128, 283-288, 2006.

20. Roux, D., A. Vian, S. Girard, P. Bonnet, F. Paladian, E. Davies, and G. Ledoigt, "High frequency $(900 \mathrm{MHz})$ low amplitude $\left(5 \mathrm{~V} \cdot \mathrm{m}^{-1}\right)$ electromagnetic field: A genuine environmental stimulus that affects transcription, translation, calcium and energy charge in tomato," Planta, Vol. 227, 883-891, 2008. 
21. "Reverberation chamber test method," IEC Draft 61000-4-21, Electromagnetic Compatibility (EMC), Part 4: Testing and Measure Techniques, Section 21, 2000.

22. "Radio frequency susceptibility (radiated and conducted)," RTCA/DO-160D Environmental Conditions and Test Procedures for Airborne Equipment, Draft \#8, Section 20, 2000.

23. Corona, P., J. Ladbury, and G. Latmiral, "Reverberation-chamber research — Then and now: A review of early work and comparison with current understanding," IEEE Transactions on Electromagnetic Compatibility, Vol. 44, 87-94, 2002.

24. D'Ambrosio, G., F. Di Meglio, and G. Ferrara, "Multimode timevarying enclosures for exposure and dosimetry in bioelectromagnetic experiments," Alta Frequenza, Vol. 49, 89-94, 1980.

25. Corona, P., G. Ferrara, and M. Migliaccio, "Reverberation chambers as sources of stochastic electromagnetic fields," IEEE Transactions on Electromagnetic Compatibility, Vol. 38, No. 3, 348-356, August 1996.

26. Bruns, C., "Three-dimensional simulation and experimental verification of a reverberation chamber," Doctoral Thesis, Swiss Federal Institute of Technology, Zurich, 2005.

27. Lunden, O. and M. Backstrom, "Stirrer efficiency in FOA reverberation chambers. Evaluation of correlation coefficients and chi-squared tests," IEEE International and Symposium on Electromagnetic Compatibility, Vol. 1, 11-16, Washington, 2000.

28. Dawson, J. F., "Reverberation (mode-stirred) chambers for electromagnetic compatibility," EMC and Compliance, Vol. 46, 30-28, 2003.

29. Holloway, C., D. Hill, J. Ladbury, P. Wilson, G. Koepke, and J. Coder, "On the use of reverberation chambers to simulate a Rician radio environment for the testing of wireless devices," IEEE Trans. on Ant. and Prop., Vol. 54, No. 11, November 2006.

30. Hill, D. A., "Plane wave integral representation for fields in reverberation chambers," IEEE Transactions on Electromagnetic Compatibility, Vol. 40, No. 3, 209-217, 1998.

31. "Micropuce à ADN pour études physiologiques," Grant from the French Foundation for Health and Radiofrequencies (Fondation Santé et Radiofréquences), 2009, http://www.santeradiofrequences.org.

32. Lovisolo G. A., F. Apollonio, L. Ardoino, M. Liberti, V. Lopresto, C. Marino, A. Paffi, and R. Pinto, "Specifications of in vitro exposure setups in the radio-frequency range," Radio Science 
Bulletin, No. 331, 21-30, December 2009.

33. Massey, F. J., "The Kolmogorov-Smirnov test for goodness of fit," Journal of the American Statistical Association, Vol. 46, 68-78, March 1951.

34. Taflove, A. and S. C. Hagness, Computational Electrodynamics, 2nd Edition, Artech House, 2000.

35. Collin, A., "Dosimétrie de systèmes d'exposition pour l'étude in vivo ou in vitro des interactions des ondes électromagnétiques décimétriques et centimétriques avec le vivant," Doctoral Thesis, Limoges University, 2007.

36. Bonnet, P., F. Diouf, C. Chauvière, S. Lalléchère, M. Fogli, and F. Paladian, "Numerical simulation of a reverberation chamber with a stochastic collocation method," Compte Rendu de l'Académie des Sciences/Physique, 2009, doi:10.1016/j.crhy.2008.12.001.

37. Ticaud, N., C. Merla, D. Arnaud-Cormos, and P. Lévêque, "Caractérisations numérique et expérimentale d'un système pour l'exposition en temps réel de neurones aux ondes radiofréquence," 15th International Symposium CEM210, Limoges, France, April 2010. 